\title{
THE PROPERTIES OF CONVOLUTION TYPE TRANSFORMS IN WEIGHTED ORLICZ SPACES
}

\author{
Yunus E. YiLdiRIR AND DaniYal M. IsRafilov \\ Balikesir University, Turkey
}

\begin{abstract}
In the weighted Orlicz spaces a convolution type transform is defined and a relation between this transform and the best approximation by trigonometric polynomials in the weighted Orlicz spaces is obtained.
\end{abstract}

\section{INTRODUCTION AND MAIN RESULTS}

A convex and continuous function $\varphi:[0, \infty) \rightarrow[0, \infty)$ for which $\varphi(0)=0$, $\varphi(x)>0$ for $x>0$, and

$$
\lim _{x \rightarrow 0} \frac{\varphi(x)}{x}=0, \quad \lim _{x \rightarrow \infty} \frac{\varphi(x)}{x}=\infty
$$

is called a Young function. The complementary Young function $\psi$ of $\varphi$ is defined by

$$
\psi(y):=\max \{x y-\varphi(x): x \geq 0\}
$$

for $y \geq 0$.

By $L_{p}[0,2 \pi], 1 \leq p \leq \infty$, we denote the Lebesgue space of $2 \pi$ periodic functions $f$.

Let $\varphi$ be a Young function and $\psi$ be its complementary Young function. By $L_{\varphi}[0,2 \pi]$ we denote the Orlicz space of $2 \pi$ periodic functions $f$, for which

$$
\int_{0}^{2 \pi} \varphi[|f(x)|] d x<\infty
$$

2010 Mathematics Subject Classification. 41A10, 42A10.

Key words and phrases. Convolution type transform, weighted Orlicz space, best approximation. 
with the Orlicz norm

$$
\|f\|_{\varphi}:=\sup \left\{\int_{0}^{2 \pi}|f(x) g(x)| d x: \int_{0}^{2 \pi} \psi[|g(x)|] d x \leq 1\right\}
$$

or with the Luxemburg norm

$$
\|f\|_{(\varphi)}:=\inf \left(k>0: \int_{0}^{2 \pi} \varphi\left[k^{-1}|f(x)|\right] d x \leq 1\right) .
$$

These norms make $L_{\varphi}[0,2 \pi]$ a Banach space [3, p. 69]. The Orlicz and Luxemburg norms satisfy the inequalities

$$
\|f\|_{(\varphi)} \leq\|f\|_{\varphi} \leq 2\|f\|_{(\varphi)}, \quad f \in L_{\varphi}[0,2 \pi]
$$

and so they are equivalent $[3$, p. 80]. Furthermore the Orlicz norm can be determined by means of the Luxemburg norm ([3, pp. 79-80]):

$$
\|f\|_{\varphi}:=\sup \left\{\int_{0}^{2 \pi}|f(x) g(x)| d x:\|g\|_{(\varphi)} \leq 1\right\}
$$

and Hölder's inequalities

$$
\begin{aligned}
& \int_{0}^{2 \pi}|f(x) g(x)| d x \leq\|f\|_{\varphi}\|g\|_{(\psi)} \\
& \int_{0}^{2 \pi}|f(x) g(x)| d x \leq\|f\|_{(\varphi)}\|g\|_{\psi}
\end{aligned}
$$

hold for every $f \in L_{\varphi}[0,2 \pi]$ and $g \in L_{\psi}[0,2 \pi]([3$, p. 80]).

For a quasiconvex function $\phi$, following $[2$, p. 218] we put

$$
\frac{1}{p(\phi)}:=\inf \left\{\beta: \phi^{\beta} \text { is quasiconvex }\right\} \text {. }
$$

The number $p(\phi)$ is called the index of $\phi$.

A measurable function $\omega:[0,2 \pi] \rightarrow[0, \infty]$ is called a weight function if the set $\omega^{-1}(\{0, \infty\})$ has Lebesgue measure zero.

Let $\omega$ be a weight function. If we write $\omega(x) d x$ instead of $d x$ in (1.1) we obtain the weighted Orlicz space $L_{\varphi, \omega}[0,2 \pi]$. The weighted Orlicz norm is denoted by $\|f\|_{\varphi, \omega}$ and the weighted Luxemburg norm is denoted by $\|f\|_{(\varphi, \omega)}$. These norms make $L_{\varphi, \omega}[0,2 \pi]$ a Banach space. 
Let $1<p<\infty$ and $1 / p+1 / q=1$. A weight function $\omega$ belongs to the Muckenhoupt class $A_{p}[0,2 \pi]$ if

$$
\left(\frac{1}{|I|} \int_{I} \omega^{p}(x) d x\right)^{1 / p}\left(\frac{1}{|I|} \int_{I} \omega^{-q}(x) d x\right)^{1 / q} \leq C
$$

with a finite constant $C$ independent of $I$, where $I$ is any subinterval of $[0,2 \pi]$ and $|I|$ denotes the length of $I$.

Let $L_{\varphi, \omega}[0,2 \pi]$ be a weighted Orlicz space and let $p(\varphi)$ be the index of $\varphi$. For $f \in L_{\varphi, \omega}[0,2 \pi]$ we define the operator $\sigma_{h}$ by

$$
\left(\sigma_{h} f\right)(x, u):=\frac{1}{2 h} \int_{-h}^{h} f(x+t u) d t, \quad 0<h<\pi, x \in[0, \pi], \quad-\infty<u<\infty .
$$

With respect to [2, Theorem 6.4.4, p. 250], the operator $\sigma_{h}$ is a bounded linear operator on $L_{\varphi, \omega}[0,2 \pi]$ under the conditions that $\varphi^{\alpha}$ is quasiconvex for some $\alpha, 0<\alpha<1$, and $\omega \in A_{p(\varphi)}[0,2 \pi]$.

We denote by $E_{n}(f)_{\varphi, \omega}$ the best approximation of $f \in L_{\varphi, \omega}[0,2 \pi]$ by trigonometric polynomials of degree not exceeding $n$, i.e.,

$$
E_{n}(f)_{\varphi, \omega}=\inf \left\{\left\|f-T_{n}\right\|_{\varphi, \omega}: T_{n} \in \Pi_{n}\right\},
$$

where $\Pi_{n}$ denotes the class of trigonometric polynomials of degree at most $n$. Note that the existence of $T_{n}^{*} \in \Pi_{n}$ such that

$$
E_{n}(f)_{\varphi, \omega}=\left\|f-T_{n}^{*}\right\|_{\varphi, \omega}
$$

follows, for example, from [1, Theorem 1.1, p. 59].

The convolution type transforms play an important role in the many areas of theoretical and applied mathematics. In particular, these objects are very useful in the approximation theory for the constructions of the approximating polynomials. Therefore, it is necessary to study the relation between these transforms and the best approximations numbers $E_{n}(f)_{\varphi, \omega}$ in the weighted Orlicz spaces $L_{\varphi, \omega}[0,2 \pi]$. In the nonweighted Orlicz spaces these transforms are constructed by using the usual shift $f(x-h u)$ for a given function $f \in$ $L_{\varphi}[0,2 \pi]$. But the weighted Orlicz spaces are noninvariant with respect to the usual shift $f(x-h u)$. Therefore, we define the convolution type transforms by using the mean value function $\left(\sigma_{h} f\right)(x, u)$, defined above.

In the weighted Orlicz space $L_{\varphi, \omega}[0,2 \pi]$ we define

$$
D(f, \sigma, h, \varphi):=\left\|\int_{-\infty}^{\infty}\left(\sigma_{h} f\right)(., u) d \sigma(u)\right\|_{\varphi, \omega}, \quad f(x) \in L_{\varphi, \omega}[0,2 \pi],
$$


where $\sigma(u)$ is a real function of bounded variation on the real axis $-\infty<u<$ $\infty$. It will be assumed that

$$
\int_{-\infty}^{\infty} d \sigma(u)=0
$$

Throughout this paper, the constant $c$ denotes a generic constant, i.e. a constant whose values can change even between different occurrences in a chain of inequalities.

The following theorem estimates the quantity $D(f, \sigma, h, \varphi)$ in terms of the best trigonometric approximation of the function $f$ in the weighted Orlicz spaces.

TheOREM 1.1. Let $L_{\varphi, \omega}[0,2 \pi]$ be reflexive, $f \in L_{\varphi, \omega}[0,2 \pi], \omega \in$ $A_{p(\varphi)}[0,2 \pi]$ and $\varphi^{\alpha}$ be quasiconvex for some $\alpha, 0<\alpha<1$, such that

$$
\varphi(u v) \leq c \varphi(u) \varphi(v)
$$

with a constant $c>0$. Then for every natural number $m$

$$
D(f, \sigma, h, \varphi) \leq c\left(\sum_{r=0}^{m} E_{2^{r}-1}^{2}(f)_{\varphi, \omega} \cdot \delta_{2^{r}, h}^{2}\right)^{1 / 2}+c E_{2^{m+1}}(f)_{\varphi, \omega}
$$

if $\varphi(\sqrt{u})$ is convex and

$$
\begin{gathered}
D(f, \sigma, h, \varphi) \leq \operatorname{cinf}_{k>0} k^{-1}\left(1+\sum_{r=0}^{m} c \varphi\left(k E_{2^{r}-1}(f)_{\varphi, \omega} \cdot \delta_{2^{r}, h}\right)\right) \\
+c E_{2^{m+1}}(f)_{\varphi, \omega}
\end{gathered}
$$

if $\varphi(\sqrt{u})$ is concave, where

$$
\begin{aligned}
& \delta_{2^{r}, h}:=\sum_{l=2^{r}}^{2^{r+1}-1}|\hat{\sigma}(l h)-\hat{\sigma}((l+1) h)|+\left|\hat{\sigma}\left(2^{r} h\right)\right|, \\
& \hat{\sigma}(x):=\int_{-\infty}^{\infty} \frac{\sin u x}{u x} d \sigma(u), \quad 0<h \leq \pi .
\end{aligned}
$$

In spite of the fact that the condition (1.3) is rather strong, there are many nontrivial examples of functions satisfying this condition. For example, the function $\varphi(x)=\log (1+x), x>0$ satisfies this condition (see, also, [8, pp. 28-34]).

TheOREM 1.2. Let $L_{\varphi, \omega}[0,2 \pi]$ be reflexive, $f \in L_{\varphi, \omega}[0,2 \pi], \omega \in$ $A_{p(\varphi)}[0,2 \pi]$ and $\varphi^{\alpha}$ be quasiconvex for some $\alpha, 0<\alpha<1$ such that

$$
\varphi(u v) \leq c \varphi(u) \varphi(v)
$$


with a constant $c>0$. Let $F(x)$ be a bounded variation function, i.e.,

$$
\|F(x)\| \leq c_{1}, \quad \sum_{\theta=2^{\mu}}^{2^{\mu+1}-1}|F(\theta h)-F((\theta+1) h)| \leq c_{2}, h \leq 2^{-m-1} .
$$

If $\sigma_{1}$ and $\sigma_{2}$ are the functions satisfying the condition

$$
\hat{\sigma}_{1}(x)=\hat{\sigma}_{2}(x) F(x), \quad|x|<1
$$

then

$$
D\left(f, \sigma_{1}, h, \varphi\right)=c\left[D\left(f, \sigma_{2}, h, \varphi\right)+E_{2^{m+1}}(f)_{\varphi, \omega}\right] .
$$

The unweighted versions of these theorems, when the convolution type transform is defined with respect to the usual shift $f(x-h u)$, instead of $\sigma(h f)(x)$, were proved in [6].

\section{Auxiliary Result}

The following Lemma is known as Marcinkiewicz Interpolation Theorem on quasi-linear operators ([7, p. 193]):

Lemma 2.1. Suppose that a quasi-linear operator $T$ is simultaneously of weak types $(\alpha, \alpha)$ and $(\beta, \beta)$ where $1 \leq \alpha<\beta<\infty, \mu(\Omega)<\infty$. If $L_{\phi}(\mu)$ is reflexive and

$$
\begin{aligned}
& \int_{u}^{\infty} \frac{\phi(t)}{t^{\beta+1}} d t=O\left\{\frac{\phi(u)}{u^{\beta}}\right\}, \\
& \int_{0}^{u} \frac{\phi(t)}{t^{\alpha+1}} d t=O\left\{\frac{\phi(u)}{u^{\alpha}}\right\},
\end{aligned}
$$

then $g:=T f, f \in L_{\phi}(\mu)$, is defined and satisfies the inequality

$$
\int_{\Omega} \phi(T f) d \mu \leq K\left(\int_{\Omega} \phi(f) d \mu+1\right),
$$

for some $K$ independent of $f$, where $(\Omega, \Sigma, \mu)$ is measure space on which $L_{\phi}(\mu)$ is defined.

Then

Lemma 2.2. Let $L_{\varphi, \omega}[0,2 \pi]$ be reflexive, $f \in L_{\varphi, \omega}[0,2 \pi], \omega \in A_{p(\varphi)}[0,2 \pi]$.

$$
c\left\|\left(\sum_{\mu=1}^{\infty}\left|\Delta_{\mu}\right|^{2}\right)^{1 / 2}\right\|_{\varphi, \omega} \leq\|f\|_{\varphi, \omega} \leq C\left\|\left(\sum_{\mu=1}^{\infty}\left|\Delta_{\mu}\right|^{2}\right)^{1 / 2}\right\|_{\varphi, \omega}
$$


with the constants $c$ and $C$ independent of $f$, where

$$
\Delta_{\mu}:=\Delta_{\mu}(x, f):=\sum_{\nu=2^{\mu-1}}^{2^{\mu}-1} c_{\nu} e^{i \nu x} .
$$

Proof. Let $f \in L_{\varphi, \omega}[0,2 \pi]$ and the series

$$
f(x) \sim \sum_{k=-\infty}^{\infty} c_{k} e^{i k x}
$$

be its Fourier series with $c_{0}=0$. By [4] for $f \in L_{p, \omega}[0,2 \pi], p>1$, there are constants $E, F$ independent of $f$ such that

$$
\left.\left.E \int_{0}^{2 \pi}\left|\sum_{\mu=1}^{\infty}\right| \Delta_{\mu}\right|^{2}\right|^{p / 2} \omega(x) d x \leq \int_{0}^{2 \pi}|f(x)|^{p} \omega(x) d x \leq\left.\left. F \int_{0}^{2 \pi}\left|\sum_{\mu=1}^{\infty}\right| \Delta_{\mu}\right|^{2}\right|^{p / 2} \omega(x) d x .
$$

Since $L_{\varphi, \omega}[0,2 \pi]$ is reflexive, following the proof of [7, Theorem 7, p. 193] we can find numbers $\alpha, \beta, a, b$ with $1<\alpha<a<b<\beta<\infty$ and a $N$-function $\varphi_{1}$, equivalent to $\varphi$, such that

$$
\begin{aligned}
& \int_{u}^{\infty} \frac{\varphi_{1}(t)}{t^{\beta+1}} d t \leq \frac{1}{\beta-b}\left\{\frac{\varphi_{1}(u)}{u^{\beta}}\right\}, \\
& \int_{0}^{u} \frac{\varphi_{1}(t)}{t^{\alpha+1}} d t \leq \frac{1}{a-\alpha}\left\{\frac{\varphi_{1}(u)}{u^{\alpha}}\right\} .
\end{aligned}
$$

On the base of (2.2) we define a quasi-linear operator

$$
T f(x):=\left(\sum_{\mu=1}^{\infty}\left|\Delta_{\mu}(x, f)\right|^{2}\right)^{1 / 2}
$$

which is bounded (in particular is of weak type $(p, p)$ ) in $L_{p, \omega}[0,2 \pi]$ for every $p>1$ by (2.3). Therefore the hypothesis of Lemma 2.1 fulfills. For $d \mu=$ $\omega(x) d x$ in Lemma 2.1, there exists $K>1 / 2$ such that

$$
\begin{aligned}
& \int_{0}^{2 \pi} \varphi_{1}\left(\left(\sum_{\mu=1}^{\infty}\left|\Delta_{\mu}\right|^{2}\right)^{1 / 2}\right) \omega(x) d x \leq K\left(\int_{0}^{2 \pi} \varphi_{1}(|f(x)|) \omega(x) d x+1\right) . \\
& \text { If }\|f\|_{\left(\varphi_{1}, \omega\right)}=1 \text {, then }
\end{aligned}
$$

$$
\int_{0}^{2 \pi} \varphi_{1}(|f(x)|) \omega(x) d x \leq 1 .
$$


Hence we get

$$
\begin{aligned}
\int_{0}^{2 \pi} \varphi_{1}\left(\frac{1}{2 K}\left(\sum_{\mu=1}^{\infty}\left|\Delta_{\mu}\right|^{2}\right)^{1 / 2}\right) \omega(x) d x & \leq \frac{1}{2 K} \int_{0}^{2 \pi} \varphi_{1}\left(\left(\sum_{\mu=1}^{\infty}\left|\Delta_{\mu}\right|^{2}\right)^{1 / 2}\right) \omega(x) d x \\
& \leq \frac{1}{2}\left(\int_{0}^{2 \pi} \varphi_{1}(|f(x)|) \omega(x) d x+1\right) \leq 1
\end{aligned}
$$

and if $\|f\|_{\left(\varphi_{1}, \omega\right)}=1$, then $\|T f\|_{\left(\varphi_{1}, \omega\right)} \leq 2 K$. The last inequality implies that

$$
\left\|\left(\sum_{\mu=1}^{\infty}\left|\Delta_{\mu}\right|^{2}\right)^{1 / 2}\right\|_{\left(\varphi_{1}, \omega\right)} \leq 2 K\|f\|_{\left(\varphi_{1}, \omega\right)}
$$

and

$$
\left\|\left(\sum_{\mu=1}^{\infty}\left|\Delta_{\mu}\right|^{2}\right)^{1 / 2}\right\|_{\varphi_{1}, \omega} \leq 4 K\|f\|_{\varphi_{1}, \omega}
$$

which implies the left hand side of the required result (2.1)

$$
\left\|\left(\sum_{\mu=1}^{\infty}\left|\Delta_{\mu}\right|^{2}\right)^{1 / 2}\right\|_{\varphi, \omega} \leq C\|f\|_{\varphi, \omega} .
$$

Using Hölder's inequality for $f \in L_{\varphi, \omega}[0,2 \pi], g \in L_{\psi, \omega}[0,2 \pi],(2.5)$ and ( 1.2 ) we obtain

$$
\begin{aligned}
& \int_{0}^{2 \pi}|f(x) g(x)| \omega(x) d x \\
& =\int_{0}^{2 \pi}\left|\sum_{\mu=1}^{\infty} \Delta_{\mu}(x, f) \Delta_{\mu}(x, g)\right| \omega(x) d x \leq \int_{0}^{2 \pi} \sum_{\mu=1}^{\infty}\left|\Delta_{\mu}(x, f) \Delta_{\mu}(x, g)\right| \omega(x) d x \\
& \leq \int_{0}^{2 \pi}\left[\sum_{\mu=1}^{\infty}\left|\Delta_{\mu}(x, f)\right|^{2}\right]^{1 / 2}\left[\sum_{\mu=1}^{\infty}\left|\Delta_{\mu}(x, g)\right|^{2}\right]^{1 / 2} \omega(x) d x \\
& \leq\left\|\left[\sum_{\mu=1}^{\infty}\left|\Delta_{\mu}(x, f)\right|^{2}\right]^{1 / 2}\right\|\left\|_{\varphi, \omega}\right\|\left[\sum_{\mu=1}^{\infty}\left|\Delta_{\mu}(x, g)\right|^{2}\right]^{1 / 2} \|_{(\psi, \omega)} \\
& \leq 2 c\left\|\left[\sum_{\mu=1}^{\infty}\left|\Delta_{\mu}(x, f)\right|^{2}\right]^{1 / 2}\right\|_{\varphi, \omega}\|g\|_{(\psi, \omega)} \text {. }
\end{aligned}
$$


Now taking supremum in the last inequality for all functions $g \in L_{\psi, \omega}[0,2 \pi]$ satisfying $\|g\|_{(\psi, \omega)} \leq 1$, we find

$$
\|f\|_{\varphi, \omega} \leq C\left\|\left(\sum_{\mu=1}^{\infty}\left|\Delta_{\mu}\right|^{2}\right)^{1 / 2}\right\|_{\varphi, \omega}
$$

and the proof of Lemma 2.2 is established.

Lemma 2.3. Let $f_{n}(x)(n=1,2, \ldots)$ be a sequence of $2 \pi$ periodic functions in a reflexive Orlicz space $L_{\varphi, \omega}[0,2 \pi], \omega \in A_{p(\varphi)}[0,2 \pi]$, and let $S_{n, k_{n}}(x)$ be the $k$-th partial sum of Fourier series of the function $f_{n}(x), k=k_{n}$ is a function of $n$. Then

$$
\left\|\left(\sum_{n=1}^{\infty}\left|S_{n, k_{n}}(x)\right|^{2}\right)^{1 / 2}\right\|_{\varphi, \omega} \leq C\left\|\left(\sum_{n=1}^{\infty}\left|f_{n}(x)\right|^{2}\right)^{1 / 2}\right\|_{\varphi, \omega}
$$

with a constant $C$ is independent of $f_{n}(x)$.

ProOF. For

$$
f(x):=\left(\sum_{n=1}^{\infty}\left|f_{n}(x)\right|^{2}\right)^{1 / 2}
$$

we define the quasilinear operator

$$
T f(x):=\left(\sum_{n=1}^{\infty}\left|S_{n, k_{n}}(x)\right|^{2}\right)^{1 / 2},
$$

which is bounded (in particular is of weak type $(p, p)$ ) in $L_{p}[0,2 \pi]$ for every $p>1$ by [5] (see, also, [9] and [11, p. 225]). Now, the required inequality is obtained by applying Lemma 2.1 and by repeating afterwards step by step the proof of the left hand side of Lemma 2.2.

Lemma 2.4. Let $\omega \in A_{p(\varphi)}[0,2 \pi]$ and $\lambda_{0}, \lambda_{1}, \ldots$ be a sequence of numbers such that

$$
\left|\lambda_{l}\right| \leq M, \quad \sum_{\nu=2^{l}}^{2^{l+1}-1}\left|\lambda_{\nu}-\lambda_{\nu+1}\right| \leq M \quad(l=0,1,2, \ldots) .
$$

Then the series $a_{0} \lambda_{0} / 2+\sum_{\nu=0}^{\infty} \lambda_{\nu}\left(a_{\nu} \cos \nu x+b_{\nu} \sin \nu x\right)$, where $a_{\nu}, b_{\nu}$ are the Fourier coefficients of a function $f \in L_{\varphi, \omega}[0,2 \pi]$, is a Fourier series of some function $h \in L_{\varphi, \omega}[0,2 \pi]$ and the following inequality is valid:

$$
\int_{0}^{2 \pi} \varphi(|h(x)|) \omega(x) d x \leq C \int_{0}^{2 \pi} \varphi(|f(x)|) \omega(x) d x .
$$


Proof. We let $\Delta_{\mu, s}:=\sum_{\nu=2^{\mu-1}}^{s} A_{\nu}(x), A_{\nu}(x):=a_{\nu} \cos \nu x+b_{\nu} \sin \nu x$ $\left(s \geq 2^{\mu-1} ; \mu=1,2, \ldots\right), \Delta_{\mu}^{\prime}:=\sum_{\nu=2^{\mu-1}}^{2^{\mu}-1} \lambda_{\nu} A_{\nu}(x)$. Then, as in [10, p. 347], we obtain

$$
\left|\Delta_{\mu}^{\prime}\right|^{2} \leq 2 M\left(\sum_{s=2^{\mu-1}}^{2^{\mu}-1}\left|\Delta_{\mu, s}\right|^{2}\left|\lambda_{s}-\lambda_{s+1}\right|+\left|\Delta_{\mu}\right|^{2}\left|\lambda_{2^{\mu}}\right|\right) .
$$

Hence, according to Lemma 2.3 and (2.6)

$$
\begin{aligned}
& \int_{0}^{2 \pi} \varphi\left(\left(\sum_{\mu=1}^{\infty}\left|\Delta_{\mu}^{\prime}\right|^{2}\right)^{1 / 2}\right) \omega(x) d x \\
& \leq \int_{0}^{2 \pi} \varphi\left((2 M)^{1 / 2}\left(\sum_{\mu=1}^{\infty}\left(\sum_{s=2^{\mu-1}}^{2^{\mu}-1}\left|\Delta_{\mu, s}\right|^{2}\left|\lambda_{s}-\lambda_{s+1}\right|+\left|\Delta_{\mu}\right|^{2}\left|\lambda_{2^{\mu}}\right|\right)\right)^{1 / 2}\right) \omega(x) d x \\
& \leq C \int_{0}^{2 \pi} \varphi\left((2 M)^{1 / 2}\left(\sum_{\mu=1}^{\infty}\left|\Delta_{\mu}\right|^{2}\left(\sum_{s=2^{\mu-1}}^{2^{\mu}-1}\left|\lambda_{s}-\lambda_{s+1}\right|+\left|\lambda_{2^{\mu}}\right|\right)\right)^{1 / 2}\right) \omega(x) d x \\
& \leq C \int_{0}^{2 \pi} \varphi\left(2 M\left(\sum_{\mu=1}^{\infty}\left|\Delta_{\mu}\right|^{2}\right)^{1 / 2}\right) \omega(x) d x .
\end{aligned}
$$

The inequality (2.7) follows from Lemma 2.3.

\section{Proofs of main Results}

Proof of Theorem 1.1. Let $f \in L_{\varphi, \omega}[0,2 \pi]$ and $S_{2^{m+1}}$ be the partial sum of its Fourier series and $h \leq 2^{-m-1}$. By virtue of the definition of the number $D(f, \sigma, h, \varphi)$ and the properties of the norm we have

$$
\begin{aligned}
D(f, \sigma, h, \varphi) & =\left\|\int_{-\infty}^{\infty}\left(\sigma_{h} f\right)(x) d \sigma(u)\right\|_{\varphi, \omega} \\
\leq & \left\|\int_{-\infty}^{\infty}\left[\left(\sigma_{h} f\right)(x)-\left(\sigma_{h} S_{2^{m+1}}\right)(x)\right] d \sigma(u)\right\|_{\varphi, \omega} \\
& +\left\|\int_{-\infty}^{\infty}\left(\sigma_{h} S_{2^{m+1}}\right)(x) d \sigma(u)\right\|_{\varphi, \omega} .
\end{aligned}
$$

Using $[2$, Theorem 6.7.1, p. 278], we get

$$
\left\|f(x)-S_{n}(f, x)\right\|_{\varphi, \omega} \leq c(\varphi) E_{n}(f)_{\varphi, \omega} .
$$


Considering the properties of $\sigma(u)$ and (3.1), we have

$$
D(f, \sigma, h, \varphi) \leq\left\|\int_{-\infty}^{\infty}\left(\sigma_{h} S_{2^{m+1}}\right)(x) d \sigma(u)\right\|_{\varphi, \omega}+c(\varphi, \sigma) E_{2^{m+1}}(f)_{\varphi, \omega} .
$$

Without loss of generality we suppose that Fourier series of $f(x)$ is

$$
\sum_{r=1}^{\infty} c_{r} e^{i r x}=\sum_{r=1}^{\infty} A_{r}(x)
$$

Then

$$
\begin{aligned}
\int_{-\infty}^{\infty}\left(\sigma_{h} S_{2^{m+1}}\right)(x) d \sigma(u)=\int_{-\infty}^{\infty}\left(\frac{1}{2 h} \int_{-h}^{h} S_{2^{m+1}}(x+t u) d t\right) d \sigma(u) \\
=\int_{-\infty}^{\infty}\left(\frac{1}{2 h} \int_{-h}^{h} \sum_{r=1}^{2^{m+1}-1} c_{r} e^{i r(x+t u)} d t\right) d \sigma(u) \\
=\int_{-\infty}^{\infty}\left(\frac{1}{2 h} \sum_{r=1}^{2^{m+1}-1} c_{r} e^{i r x} \int_{-h}^{h} e^{i r t u} d t\right) d \sigma(u) \\
=\sum_{r=1}^{2^{m+1}-1} A_{r}(x) \int_{-\infty}^{\infty} \frac{e^{i r h u}-e^{-i r h u}}{2 i r h u} d \sigma(u)=\sum_{r=1}^{2^{m+1}-1} A_{r}(x) \hat{\sigma}(r h) .
\end{aligned}
$$

Therefore

$$
D(f, \sigma, h, \varphi) \leq\left\|\sum_{r=1}^{2^{m+1}-1} A_{r}(x) \hat{\sigma}(r h)\right\|_{\varphi, \omega}+c E_{2^{m+1}}(f)_{\varphi, \omega} .
$$

From Lemma 2.2 and (1.2), we obtain

$$
\begin{gathered}
\left\|\sum_{r=1}^{2^{m+1}-1} A_{r}(x) \hat{\sigma}(r h)\right\|_{\varphi, \omega} \leq C\left\|\left(\sum_{r=0}^{m}\left|\sum_{l=2^{r}}^{2^{r+1}-1} A_{l}(x) \hat{\sigma}(l h)\right|^{2}\right)^{1 / 2}\right\|_{\varphi, \omega} \\
\leq 2 C \|\left(\left.\sum_{r=0}^{m}\left|\sum_{l=2^{r}}^{2^{r+1}-1} A_{l}(x) \hat{\sigma}(l h)\right|^{2}\right|^{1 / 2} \|\right. \\
=2 C \inf \left(k>0: \int_{0}^{2 \pi} \varphi\left(k^{-1}\left(\sum_{r=0}^{m} \Delta_{r, \sigma}^{2}\right)^{1 / 2}\right) \omega(x) d x \leq 1\right)
\end{gathered}
$$


where

$$
\Delta_{r, \sigma}:=\sum_{l=2^{r}}^{2^{r+1}-1} A_{l}(x) \hat{\sigma}(l h) .
$$

Let $\varphi(\sqrt{u})$ be convex and let's define $\Psi(u):=\varphi(\sqrt{u})$. By virtue of the properties of the norm

$$
\begin{aligned}
& \left\|\sum_{r=1}^{2^{m+1}-1} A_{r}(x) \hat{\sigma}(r h)\right\|_{\varphi, \omega} \\
& \leq 2 C \inf \left(k>0: \int_{0}^{2 \pi} \Psi\left(k^{-2} \sum_{r=0}^{m} \Delta_{r, \sigma}^{2}\right) \omega(x) d x \leq 1\right) \\
& \leq 2 C \inf \left(t^{1 / 2}>0: \int_{0}^{2 \pi} \Psi\left(t^{-1}\left(\sum_{r=0}^{m} \Delta_{r, \sigma}^{2}\right)\right) \omega(x) d x \leq 1\right) \\
& \leq 2 C\left\|\sum_{r=0}^{m} \Delta_{r, \sigma}^{2}\right\|_{(\Psi, \omega)}^{1 / 2} \leq 2 C\left(\sum_{r=0}^{m}\left\|\Delta_{r, \sigma}^{2}\right\|_{(\Psi, \omega)}\right)^{1 / 2} \\
& =2 C\left(\sum_{r=0}^{m}\left\|\Delta_{r, \sigma}\right\|_{(\varphi, \omega)}^{2}\right)^{1 / 2},
\end{aligned}
$$

because

$$
\begin{aligned}
\left\|\Delta_{r, \sigma}^{2}\right\|_{(\Psi, \omega)} & =\inf \left(k>0: \int_{0}^{2 \pi} \Psi\left(k^{-1} \Delta_{r, \sigma}^{2}\right) \omega(x) d x \leq 1\right) \\
& =\inf \left(k>0: \int_{0}^{2 \pi} \varphi\left(k^{-1 / 2} \Delta_{r, \sigma}\right) \omega(x) d x \leq 1\right) \\
& =\inf \left(t^{2}>0: \int_{0}^{2 \pi} \varphi\left(t^{-1} \Delta_{r, \sigma}\right) \omega(x) d x \leq 1\right) \\
& =\left\|\Delta_{r, \sigma}\right\|_{(\varphi, \omega)}^{2} .
\end{aligned}
$$

Applying the Abel transform to $\Delta_{r, \sigma}$, we obtain

$$
\begin{aligned}
\Delta_{r, \sigma}= & \sum_{l=2^{r}}^{2^{r+1}-1}\left[S_{l}(f, x)-S_{2^{r+1}-1}(f, x)\right][\hat{\sigma}(l h)-\hat{\sigma}((l+1) h)] \\
& +\left[S_{2^{r+1}-1}(f, x)-S_{2^{r}-1}(f, x)\right] \hat{\sigma}\left(2^{r} h\right) .
\end{aligned}
$$


From (3.1) and (1.2)

$$
\begin{aligned}
\left\|\Delta_{r, \sigma}\right\|_{(\varphi, \omega)} \leq & \sum_{l=2^{r}}^{2^{r+1}-1}\left\|S_{l}(f, x)-S_{2^{r+1}-1}(f, x)\right\|_{(\varphi, \omega)}|\hat{\sigma}(l h)-\hat{\sigma}((l+1) h)| \\
& +\left\|S_{2^{r+1}-1}(f, x)-S_{2^{r}-1}(f, x)\right\|_{(\varphi, \omega)}\left|\hat{\sigma}\left(2^{r} h\right)\right| \\
\leq & c E_{2^{r}-1}(f)_{\varphi, \omega} \delta_{2^{r}, h} .
\end{aligned}
$$

Then

$$
\left\|\sum_{r=1}^{2^{m+1}-1} A_{r}(x) \hat{\sigma}(r h)\right\|_{\varphi, \omega} \leq c\left(\sum_{r=0}^{m} E_{2^{r}-1}^{2}(f)_{\varphi, \omega} \cdot \delta_{2^{r}, h}^{2}\right)^{1 / 2}
$$

This inequality yields (1.4) by (3.4).

Let $\varphi(\sqrt{u})$ be concave. By Lemma 2.2 and [3, Theorem 10.5, p. 92],

$$
\begin{aligned}
& \left\|\sum_{r=1}^{2^{m+1}-1} A_{r}(x) \hat{\sigma}(r h)\right\|_{\varphi, \omega} \|\left(\left.\sum_{r=0}^{m}\left|\sum_{l=2^{r}}^{2^{r+1}-1} A_{l}(x) \hat{\sigma}(l h)\right|^{2}\right|^{1 / 2} \|\right. \\
& =\left.C\right|_{\varphi, \omega} \\
& =C \inf _{k>0} k^{-1}\left(1+\int_{0}^{2 \pi} \varphi\left(k\left(\sum_{r=0}^{m}\left|\sum_{l=2^{r}}^{2^{r+1}-1} A_{l}(x) \hat{\sigma}(l h)\right|^{2}\right) \omega(x) d x\right)\right. \\
& \leq C \inf _{k>0} k^{-1}\left(1+\int_{0}^{2 \pi} \varphi\left(\left(\sum_{r=0}^{m} k^{2} \Delta_{r, \sigma}^{2}\right)^{1 / 2}\right) \omega(x) d x\right) .
\end{aligned}
$$

Since $\varphi(\sqrt{u})$ is concave

$$
\left\|\sum_{r=1}^{2^{m+1}-1} A_{r}(x) \hat{\sigma}(r h)\right\|_{\varphi, \omega} \leq \inf _{k>0} k^{-1}\left(1+\sum_{r=0}^{m} \int_{0}^{2 \pi} \varphi\left(k \Delta_{r, \sigma}\right) \omega(x) d x\right) .
$$

Using the proof of $[3$, Lemma 9.2, p. 74$]$, it is easily seen that

$$
\int_{0}^{2 \pi} \varphi\left[\frac{u(x)}{\|u(x)\|_{\varphi, \omega}}\right] \omega(x) d x \leq 1 .
$$


By this inequality and (1.3)

$$
\begin{aligned}
\int_{0}^{2 \pi} \varphi\left(k \Delta_{r, \sigma}\right) \omega(x) d x & =c \int_{0}^{2 \pi} \varphi\left(\frac{\Delta_{r, \sigma}}{\left\|\Delta_{r, \sigma}\right\|_{\varphi, \omega}}\right) \varphi\left(k\left\|\Delta_{r, \sigma}\right\|_{\varphi, \omega}\right) \omega(x) d x \\
& \leq c \varphi\left(k\left\|\Delta_{r, \sigma}\right\|_{\varphi, \omega}\right) .
\end{aligned}
$$

Consequently, we obtain

$$
S(\sigma, h, \varphi) \leq C \inf _{k>0} k^{-1}\left(1+\sum_{r=0}^{m} c \varphi\left(k E_{2^{r}-1}(f)_{\varphi, \omega} \delta_{2^{r}, h}\right)\right) .
$$

This yields (1.5) by (3.4).

Proof of Theorem 1.2. Since $f \in L_{\varphi, \omega}[0,2 \pi]$ from the properties of the norm and (3.1)

$$
D\left(f, \sigma_{1}, h, \varphi\right) \leq\left\|\int_{-\infty}^{\infty}\left(\sigma_{h} S_{2^{m+1}}\right)(x) d \sigma_{1}(u)\right\|_{\varphi, \omega}+c E_{2^{m+1}}(f)_{\varphi, \omega} .
$$

Using the properties of the function $F(x)=\hat{\sigma}_{1}(x)\left(\hat{\sigma}_{2}(x)\right)^{-1},(3.3)$, Lemma 2.4 and $[2$, Theorem 6.7.1, p. 278] we obtain

$$
\begin{aligned}
& \left\|\int_{-\infty}^{\infty}\left(\sigma_{h} S_{2^{m+1}}\right)(x) d \sigma_{1}(u)\right\|_{\varphi, \omega}=\left\|\sum_{r=1}^{2^{m+1}-1} c_{r} e^{i r x} \hat{\sigma}_{2}(r h) F(r h)\right\|_{\varphi, \omega} \\
& \leq c\left\|\sum_{r=1}^{2^{m+1}-1} c_{r} e^{i r x} \hat{\sigma}_{2}(r h)\right\|_{\varphi, \omega}=c\left\|\int_{-\infty}^{\infty}\left(\sigma_{h} S_{2^{m+1}}\right)(x) d \sigma_{2}(u)\right\|_{\varphi, \omega} \\
& \quad \leq c\left\|\int_{-\infty}^{\infty} \sigma_{h} f(x) d \sigma_{2}(u)\right\|_{\varphi, \omega} .
\end{aligned}
$$

This yields (1.7) by (3.5).

\section{REFERENCES}

[1] R. A. De Vore and G. G. Lorentz, Constructive approximation, Springer, 1993.

[2] I. Genebashvili, A. Gogatishvili, V. Kokilashvili and M. Krbec, Weight theory for integral transforms on spaces of homogeneous type, Longman, 1998.

[3] M. A. Krasnosel'skiı̌, Y. B. Rutickiı̌, Convex functions and Orlicz spaces, Noordholf, 1961.

[4] D. S. Kurtz, Littlewood-Paley and multiplier theorems in weighted $L^{p}$ spaces, Trans. Amer. Math. Soc. 259 (1980), 235-254.

[5] J. Marcinkiewicz, Sur les multiplicateurs des series de Fourier, Studia Mathematica 8 (1939) 78-91. 
[6] V. G. Ponomarenko, M. F. Timan, The properties of convolution type transforms in the Orlicz spaces, in: Theory of approximation o functions, Proceedings of the institute Math. and Mech. 3, Donetsk, 1998.

[7] M. M. Rao and Z. D. Ren, Application of Orlicz spaces, Dekker, 2002.

[8] M. M. Rao and Z. D. Ren, Theory of Orlicz spaces, Dekker, 1991.

[9] G. I. Sunouchi, On the Walsh-Kaczmarz series, Proc. Amer. Math. Soc. 2 (1951), $5-11$.

[10] A. Zygmund, Trigonometric series, vol. I, Cambridge Univ. Press, Cambridge, 1959.

[11] A. Zygmund, Trigonometric series, vol. II, Cambridge Univ. Press, Cambridge, 1959.

\section{Y. E. Yildirir}

Department of Mathematics

Faculty of Education

Balikesir University

10100 Balikesir

Turkey

E-mail: yildirir@balikesir.edu.tr

\section{M. Israfilov}

Department of Mathematics

Faculty of Art and Science

Balikesir University

10145 Balikesir

Turkey

E-mail: mdaniyal@balikesir.edu.tr

Received: 2.10 .2009 .

Revised: 17.11.2009. 